The Annals of Probability

2002, Vol. 30, No. 2, 1000-1001

\title{
NOTE ABOUT "FIRST ORDER CORRECTION \\ FOR THE HYDRODYNAMIC LIMIT OF SYMMETRIC SIMPLE EXCLUSION PROCESSES WITH SPEED CHANGE IN DIMENSION $d \geq 3$ "
}

\author{
By ELISE JANVRESSE
}

Université de Rouen-CNRS UMR 6085

We refer to [1] for notation.

In [1], we proved that the first order correction for the hydrodynamic equation of symmetric simple exclusion processes with speed change in dimension $d \geq 3$ is

$$
\partial_{t} \rho^{N}=\Delta_{u}\left(\rho^{N}\left(1+\alpha \rho^{N}\right)\right)-\frac{\alpha}{N} \sum_{i, j=1}^{d} \partial_{u_{i}}^{2}\left(R_{i j}\left(\rho^{N}\right) \partial_{u_{j}} \rho^{N}\right),
$$

where $R_{i j}$ is a continuous function on $(0,1)$.

We now prove that $R=0$, which means that there is no correction of order $N^{-1}$ to the hydrodynamic limit.

Indeed, from Lemma 9.1 of [1], there exists a sequence of functions $F_{k}^{i}$ that satisfies

$$
\lim _{k \rightarrow \infty} E_{v_{m}}\left[W_{0, e_{l}} \sum_{x} \tau_{x} F_{k}^{i}\right]=-R_{i, l}(m) m(1-m),
$$

where $v_{m}$ is the product Bernoulli measure of parameter $m$ and $W_{0, e_{l}}$ is the current between 0 and $e_{l}$. Notice that $v_{m}$ is translation invariant. Therefore, since our process is gradient, we obtain

$$
E_{v_{m}}\left[W_{0, e_{l}} \sum_{x} \tau_{x} F_{k}^{i}\right]=E_{v_{m}}\left[\left(\sum_{x} \tau_{x} W_{0, e_{l}}\right) F_{k}^{i}\right]=0
$$

and thus $R_{i, l}(m)=0$.

THEOREM 1. For symmetric simple exclusion processes with speed change, under diffusive rescaling, the density of particles $q^{N}(t, u)$ at time $t$ around $u \in \mathbb{R}^{d}$ satisfies

$$
N\left(q^{N}(t, \cdot)-m(t, \cdot)\right) \rightarrow 0 \quad \text { for dimension } d \geq 3,
$$

where $m(t, u)$ is the solution of the hydrodynamic limit $\partial_{t} m=\sum_{i} \partial_{u_{i}}^{2}(m(1+\alpha m))$.

Received December 1999. 
Of course, knowing that $R=0$, one can simplify the proof of Theorem 1:

There is no need to introduce the coefficient $R$. We have to prove (see Section 3 of [1]) that $\lim _{N \rightarrow \infty} E_{N}\left[N^{1-d} \sum_{x} J(T, x / N)\left[\eta_{T}(x)-m(T, x / N)\right]\right]=0$, where $J_{0}: T^{d} \rightarrow \mathbb{R}$ is a smooth function and $J: \mathbb{R}_{+} \times T^{d} \rightarrow \mathbb{R}$ is the solution of the linear equation

$$
\partial_{s} J(s, u)+\phi^{\prime}(m(s, u)) \Delta_{u} J(s, u)=0
$$

with final condition $J(T, u)=J_{0}(u)$.

There is no need to introduce corrections of order $N^{-2}$ in the density $\psi_{t}$ appearing in the specific relative entropy (see Section 4 of [1]). Indeed, one can prove directly that the specific relative entropy $N^{-d} H_{N}(t)$ is $o\left(N^{-1}\right)$ by adding terms of the form

$$
\int_{0}^{t_{0}} d t E_{N}\left[N^{1-d} \sum_{x, i} L_{N}\left\{\alpha \partial_{u_{i}}^{2} \lambda(t, x / N) F_{i}\left(\tau_{x} \eta_{t}\right)+\left(\partial_{u_{i}} \lambda(t, x / N)\right)^{2} G_{i}\left(\tau_{x} \eta_{t}\right)\right\}\right]
$$

with $F_{i}, G_{i} \in g$, in the bound for $N^{1-d} H_{N}(t)$ obtained in Section 7 of [1].

This does not change anything since by the martingale property we have the following identity:

$$
\begin{aligned}
\int_{0}^{t_{0}} d t & E_{N}\left[N^{-d-1} \sum_{x, i} N^{2} L_{N}\left\{\partial_{u_{i}}^{2} \lambda(t, x / N) F_{i}\left(\tau_{x} \eta_{t}\right)\right\}\right] \\
= & -\int_{0}^{t_{0}} d t E_{N}\left[N^{-d-1} \sum_{x, i} \partial_{t}\left\{\partial_{u_{i}}^{2} \lambda(t, x / N) F_{i}\left(\tau_{x} \eta_{t}\right)\right\}\right] \\
& +E_{N}\left[N^{-d-1} \sum_{x, i} \partial_{u_{i}}^{2} \lambda\left(t_{0}, x / N\right) F_{i}\left(\tau_{x} \eta_{t_{0}}\right)\right] \\
& -E_{N}\left[N^{-d-1} \sum_{x, i} \partial_{u_{i}}^{2} \lambda(0, x / N) F_{i}\left(\tau_{x} \eta_{0}\right)\right]
\end{aligned}
$$

and, as $N \uparrow \infty$, the right-hand side of the last expression converges to 0 .

\section{REFERENCE}

[1] JANVRESSE, E. (1998). First order correction for the hydrodynamic limit of symmetric simple exclusion processes with speed change in dimension $d \geq 3$. Ann. Probab. 26 1874-1912.

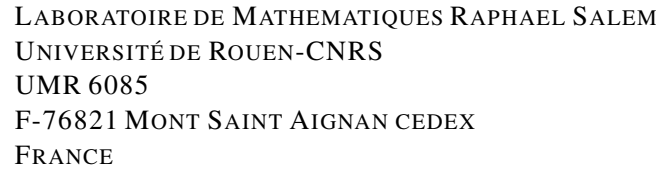

